\title{
USER-ORIENTED SYSTEMATIC OF CONTROL CONCEPTS FOR FLUID- MECHATRONIC SERVO DRIVES
}

\author{
Prof. Dr.-Ing Peter Anders ${ }^{1 *}$, Dr.-Ing Simon Ströbel ${ }^{2}$ \\ ${ }^{1}$ Hochschule Furtwangen Universit8iy, Kronenstraße 16, 78532 Tuttlingen, Germany \\ ${ }^{2}$ X-Dot Engineering, Blumenstraße 36, 72355 Schömberg, Germany \\ * Corresponding author: Tel.: +49 7461 15026620; E-mail address: an@hs-furtwangen.de
}

\begin{abstract}
This paper aims to show that controlled hydraulic drives, when properly considered and approached, are a technology that allows for a systematic and safe system design with regard to performance and energy efficiency. Controlled hydraulic drives are predestinated to be an indispensable alternative to electromechanical drives for many fields of application, especially against the background of Industry 4.0. But hydraulic drives will only be able to play this role if they see themselves as a part of mechatronics, speak the language of mechatronics and recognize the increasing importance of electric drives as part of the hydraulic toolbox as a chance.
\end{abstract}

Keywords: servo drive, mathematical model, transfer behaviour, characteristic map, model-based control, energy efficiency

\section{INTRODUCTION}

Although other topics have been more prominent in recent years, there are many quite interesting developments and trends in the field of controlled hydraulic drives. With the possibilities of digital technology available today, there are good prospects that hydraulic servo technology (at least in some application areas) will be the better alternative to electromechanical drives, so that it can maintain and even expand its current position in the world of mechatronic systems and components. The present article presents some of these developments without claiming to be complete. Due to time constraints, we will focus on the following topics in this article:

- More accurate models for controller design.

- Transparent systematics of the control concepts.

- Model-based control concepts.

- Automated model identification, optimization and tracking.

- Energy efficiency.

- Pump-directed drives / compact drives.

- Variable-speed electric drives.

- The role of computers.
The aim of this presentation is to show that hydraulic servo drives can be designed much more simply than is often claimed, especially with regard to aspects such as performance and energy efficiency. An important point here is to look at hydraulics from a more mechatronic perspective. Modern hydraulics has everything it takes to be a perfect and indispensable complement to drive solutions in the mechatronic world of Industry 4.0, especially for high load forces or special motion requirements. This article ends with some comments on what steps hydraulics should take to achieve these great prospects in the future

\section{MORE ACCURATE MODELS FOR CONTROLLER DESIGN}

\subsection{Weaknesses of conventional models}

The still most widespread and historically probably oldest type of controlled hydraulic drives is the valve-controlled hydraulic one, composed of a cylinder (or rotational motor), a constant pressure supply and a $4 / 3$ proportional directional valve. The following discussion in this chapter are mostly formulated for the cylinder drive case but can of course be directly transferred to the rotatory equivalent. 
If the traditional methods for system and controller design are often criticized as being quite fuzzy, this is certainly also due to the limited accuracy of the traditionally used steady-state models (= characteristics representing all possible steady-state operating points) and the dynamic model for controller design (= second-order transfer function), which together form the basis for system design. However, most users of these models are not aware of the background of the mathematical derivation of these models (see [1], [2] for details of this paper's chapters 2 and 3). The models are based on assumptions that one would never make in practical cases. Or, to put it more clearly: a real drive with the assumed properties would be unsuitable for real applications. The stationary model concretely is based on the assumptions that

- the servo valve has zero overlapped metering edges.

- the servo valve has linear characteristics.

- a synchronous double rod cylinder is used.

- friction is neglectable.

And for the dynamic model it is additionally assumed that

- the used piston stroke is only a small range around the neutral hydraulic centre position.

- the control valve is "infinitely fast" in relation to the dynamics of the motor itself and can therefore be dynamically regarded as a simple proportional (P-) element.

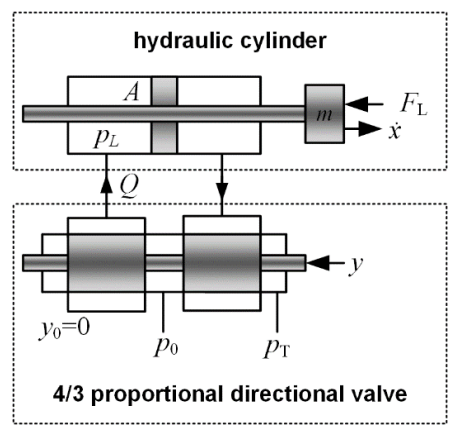

Figure 1: Simple scheme of a very idealized valvecontrolled servo drive.
However, most users of these models are not aware of the background of the mathematical derivation of these models (see [1], [2] for details of this paper's chapters 2 and 3). The models are based on assumptions that one would never make in practical cases. Or, to put it more clearly: a real drive with the assumed properties would be unsuitable for real applications.

\section{Stationary model}

For the stationary model, the relationship between the valve opening $y$ (or control signal $u$ ), load force $F_{\mathrm{L}}$ (or load pressure $p_{\mathrm{L}}$ ), and speed $\dot{x}$ (or volume flow $Q$ ) is shown in equation (1) (and equation (2) respectively) and graphically in Figure 2.

$$
\begin{gathered}
\frac{\dot{x}}{\dot{x}_{\max }}=\frac{y}{y_{\max }} \cdot \sqrt{1-\frac{F_{L}}{F_{\max }}} \\
\frac{Q}{Q_{\max }}=\frac{u}{u_{\max }} \cdot \sqrt{1-\frac{p_{L}}{p_{\max }}}
\end{gathered}
$$

A closer look reveals the consequences of these rough assumptions. As can be seen in the diagram, there is no longer a defined operating point for a very low speed or standstill at a given load. On the other hand, this is not a big surprise, as low speeds are caused by very small valve openings, but it is precisely these small openings within the negative overlap that have been neglected by the assumptions made. At higher speeds with correspondingly large valve openings, this overlap is in any case no longer significant, so that the corresponding operating points are approximately correct. This simple model is therefore suitable for rough estimations, i.e. to decide whether a concrete configuration pressure supply - valve - cylinder is sensibly dimensioned or not. The additional assumption of a symmetrical system is only approximately fulfilled for differential cylinders with a rather thin piston $\operatorname{rod}\left(\alpha=A_{\mathrm{B}} / A_{\mathrm{A}} \geq 0.7\right)$. With smaller area ratios $(\alpha<<1)$ the differences between the model-based estimated behaviour and the real behaviour become more and more significant. 


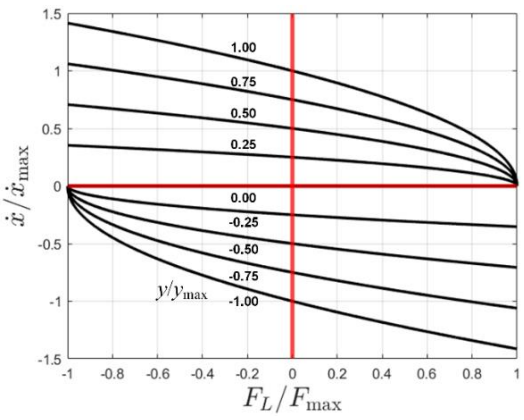

Figure 2: Static transfer behaviour of the idealized system.

\section{Dynamic model}

Concerning the dynamic transfer behaviour from the independent input signals valve opening $y$ or load force $F_{\mathrm{L}}$ to the output signal cylinder speed $\dot{x}$, the named assumptions lead to the well-known second-order transfer functions $G_{\mathrm{y}}(s)$ and $G_{\mathrm{FL}}(s)$ with the corresponding block diagram shown in Figure 3 .

$$
\begin{aligned}
& G_{y}(s)=\frac{\dot{x}(s)}{y(s)}=\frac{K \omega_{0}^{2}}{s^{2}+2 D \omega_{0} \cdot s+\omega_{0}^{2}} \\
& G_{F L}(s)=\frac{\dot{x}(s)}{F_{L}(s)}=\frac{-b_{1} \cdot s-b_{0}}{s^{2}+2 D \omega_{0} \cdot s+\omega_{0}^{2}} \\
& K=\frac{V_{Q y}}{A+\frac{V_{Q p} d_{N}}{A}} \\
& \omega_{0}=\sqrt{\frac{2}{C_{H} m}\left(A^{2}+V_{Q p} d_{N}\right)}
\end{aligned}
$$

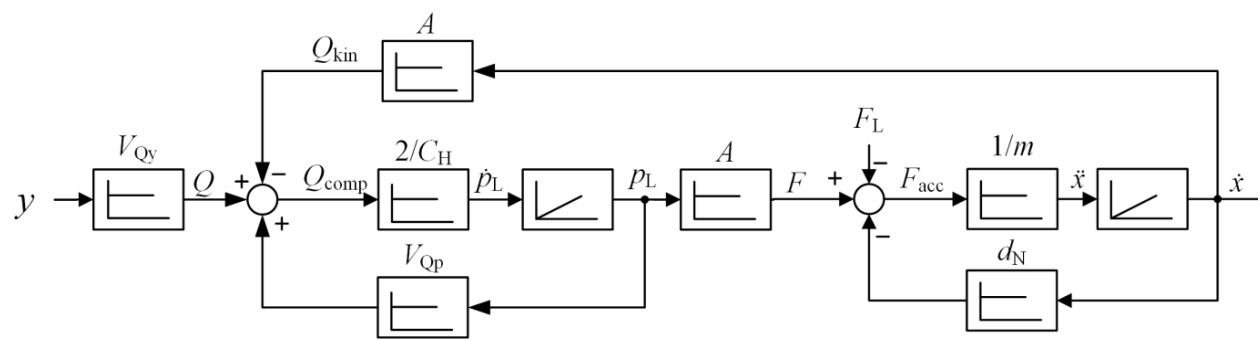

Figure 3: Block diagram of the idealized system.
$D=\frac{d_{N} \sqrt{\frac{C_{H}}{2 m}}+V_{Q p} \sqrt{\frac{2 m}{C_{H}}}}{2 \sqrt{A^{2}+V_{Q p} \cdot d_{N}}}$

$b_{1}=\frac{1}{m} \quad b_{0}=\frac{2 A V_{Q p}}{C_{H} m}$

Above all, the parameters $V_{\text {Qy }}$ and $V_{\text {Qp }}$ may be considerably influenced by the linearization of the real non-linear mathematical model equations. Therefore, they are in principal operating point dependent. These parameters result as the gradients of equation (2).

$V_{Q y}=\left.\frac{\partial Q\left(p_{L}, y\right)}{\partial y}\right|_{\mathbf{O P}}=\frac{Q_{\max }}{y_{\max }} \cdot \sqrt{1-\frac{p_{L \mathbf{O P}}}{p_{L \max }}}$

$$
V_{Q_{p}}=\left.\frac{\partial Q\left(p_{L}, y\right)}{\partial p_{L}}\right|_{\mathbf{O P}}=\frac{-Q_{\max } y_{\mathbf{O P}}}{2 p_{L \max } y_{\max }} \cdot \sqrt{1-\frac{p_{L \mathbf{O P}}}{p_{L \max }}}
$$

As in the stationary case, the erroneous assumptions in the dynamic model become immediately apparent. For example, the assumed zero overlap leads to a gradient that defines pressure gain $V_{\text {Qp }}$ to zero, i.e. the load pressure $p_{\mathrm{L}}$ would not play any role at low speeds or standstill situations, which is of course simply nonsense. Larger deviations between the theoretical model behaviour and the actually observed drive behaviour are caused by to the neglected area ratio and the center position requirement, that often isn't fulfilled in reality. The literature recommends here the application of "correction steps", i.e. via the eigenfrequency correction curve, as shown in Figure 4 and equation (11). However, practice shows that these corrections are of little help if the deviation from the 
assumptions becomes larger and especially with higher accuracy requirements.

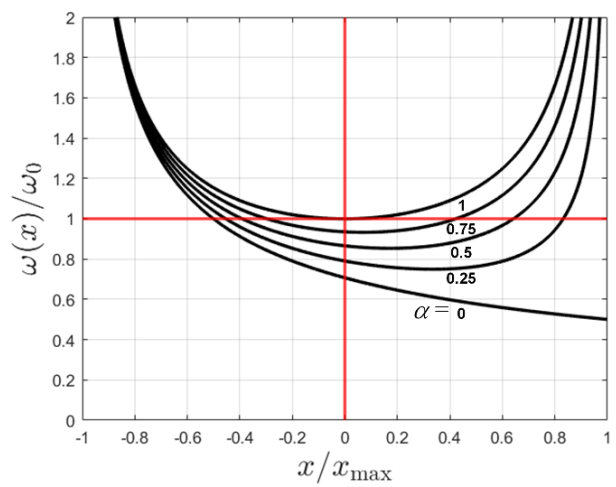

Figure 4: Eigenfrequency correction curve (dead volumes neglected).

$$
\frac{\omega(x)}{\omega_{0}}=\sqrt{\frac{x_{\max }}{2} \cdot\left(\frac{1}{x_{\max }+x+\frac{V_{\text {dead } A}}{A_{A}}}+\frac{\alpha}{x_{\max }-x+\frac{V_{\text {dead } B}}{A_{B}}}\right)}
$$

\subsection{More accurate models}

It is no surprise that controller designs based on such simple models often only show a rough coincidence between the theoretical expected and the real observed behavior. On the other hand, there are more suitable models that do not make such radical assumptions and are therefore more accurate.

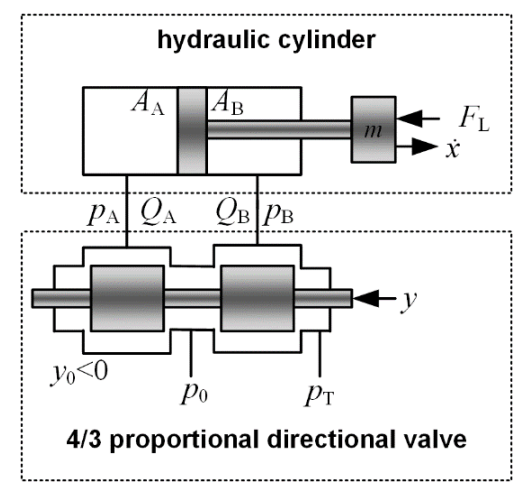

Figure 5: Simple scheme of a slightly more realistic valve-controlled servo drive.

\section{Stationary model}

A better stationary model is based on the chamber pressure diagram, which describes the pressures occurring in the cylinder chambers during stationary ( $=$ unaccelerated) motion and at standstill. With the usually available nominal data of the control valve and the cylinder, quite usable chamber pressure diagrams can be calculated.

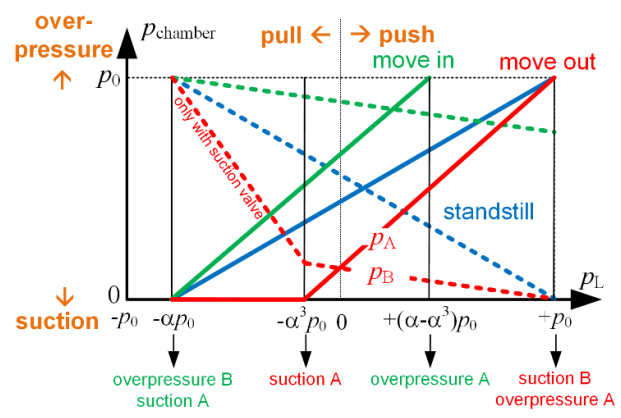

Figure 6: Pressure diagram (qualitative). Solid line $=$ chamber $\mathrm{A}$, dotted line $=$ chamber $\mathrm{B}$.

The operating point dependent volume flows and thus the speeds of the cylinder as well as the loaddependent neutral position within the overlap can also be determined via the known pressure differences across the control edges. However, this means that the complete stationary characteristic map of the drive system consisting of pressure supply-valve-cylinder is known.

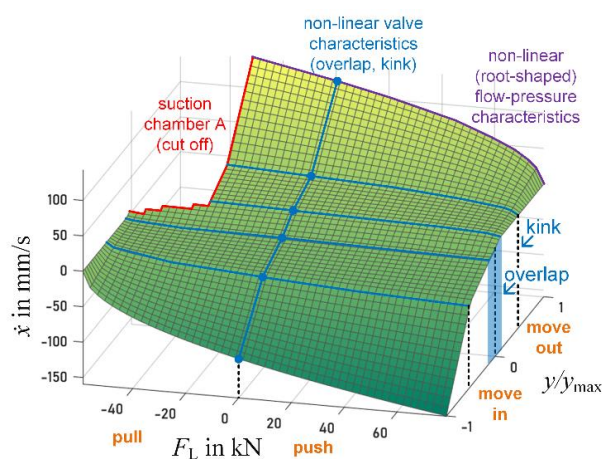

Figure 7: Characteristic map of entire system supplyvalve-cylinder, representing all reachable stationary operating points (quantitative example configuration with kinked valve characteristics and cylinder ratio $\alpha=$ $0.75)$. 
The pressure diagram or the characteristic map is also helpful to detect in advance critical operating conditions such as suction $(p<0)$ or overpressure $\left(p>p_{0}\right)$ states in a cylinder chamber due to the area ratio. The calculation shows that the dangerous suction of air in differential cylinders with pulling loads in the large chamber occurs already at a load pressure of $p_{\mathrm{L}}=-p_{0} / \alpha^{3}$. With an area ratio of e.g. $\alpha=0.5$, the maximum possible load force in the pull-in case is thus reduced to $12.5 \%$ (!) compared to the push-out case. This makes it clear how restrictive the practical effects
This model also correctly considers the area ratio and movements over the entire stroke. This model is a third-order system, as it has three integrators (two for the stroke-dependent pressure build-up and one for the movement) and six linearized parameters $V_{\mathrm{QyA}}, V_{\mathrm{QyB}}, V_{\mathrm{QpA}}, V_{\mathrm{QpB}}, C_{\mathrm{HA}}$ and $C_{\mathrm{HB}}$, see Figure 8 . The first four parameters are again derived from the non-linear valve characteristics, the last two from the piston position. Based on an operating point-dependent linearization of a real nonlinear system it is a much better description of the actual dynamic transfer behavior.

$$
G_{y}(s)=\frac{\left(\frac{A_{A} V_{Q y A}}{C_{H A} m}-\frac{A_{B} V_{Q y B}}{C_{H B} m}\right) \cdot s+\left(\frac{A_{B} V_{Q p A} V_{Q y B}-A_{A} V_{Q p B} V_{Q y A}}{C_{H A} C_{H B} m}\right)}{s^{3}+\left(\frac{V_{Q p B}}{C_{H B}}+\frac{V_{Q p A}}{C_{H A}}\right) \cdot s^{2}+\left(\frac{A_{A}^{2}}{C_{H A} m}+\frac{A_{B}^{2}}{C_{H B} m}+\frac{V_{Q p A} V_{Q p B}}{C_{H A} C_{H B}}\right) \cdot s+\left(\frac{V_{Q p B} A_{A}^{2}+V_{Q p A} A_{B}^{2}}{C_{H A} C_{H B} m}\right)}
$$

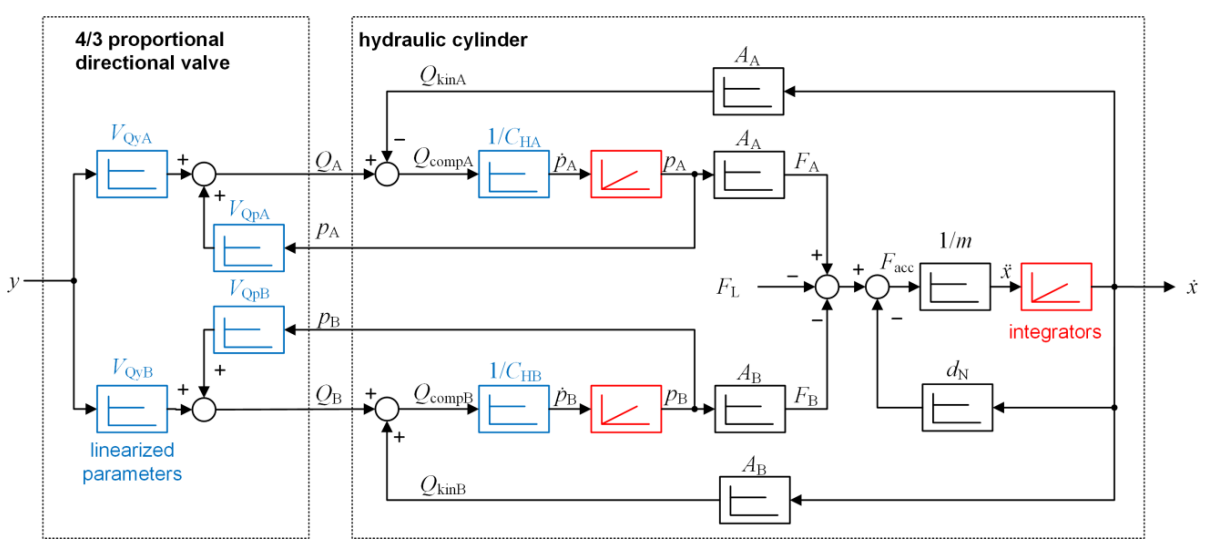

Figure 8: Linearized block diagram of more realistic system.

of the asymmetry of a cylinder drive can be.

In many cases, the real characteristic map of the specific control valve can be provided by the manufacturer on the basis of test bench measurements, which considerably simplifies the determination of a meaningful characteristic map of the overall system.

\section{Dynamic model}

A much more accurate version of the dynamic LTI model, which is important for controller design, is also available. It is obtained by describing the pressure build-up in both cylinder chambers separately and explicitly formulating the force generation over the two piston areas.
In order to apply the classical methods of control engineering, such a (linearized) LTI system description is always needed. Of course the transfer functions $G_{\mathrm{y}}(s)$ and $G_{\mathrm{FL}}(s)$ can be derived for this model, the former is given e.g. in equation (11). Even if friction is neglected in this equation, it is hardly useful to perform these calculations manually, but with the help of suitable computer algebra systems this is easily possible. Such programs can, for example, be created by the user himself with Matlab using the Control System Toolbox [3], or you can use service providers who offer such programs or perform these not quite trivial calculations.

In this context, however, the question immediately arises as to how the classic dynamic 
model as a second-order system without a zero in $G_{y}(s)$ is compatible with the expanded dynamic model as a third-order system having a zero in $G_{y}(s)$. The explanation is this: For the simple classic model, it was assumed that the piston was in the volumetric middle position (same volume in both cylinder chamber sides), but in this very constellation, a zero point and a pole position of the more accurate model become cancelled, and so in exactly this one position, the results of both models match. With increasing distance from this ideal position, the weaknesses of the simple model become apparent with the correction calculations mentioned (eigenfrequency correction curve etc.) due to increasingly significant deviations in the transfer behaviour and consequently also in the expected control behaviour.

If the position is considered as the controlled variable instead of a velocity, only one integrator has to be added in series to the output of the model, since the position is the integral of the velocity over time.
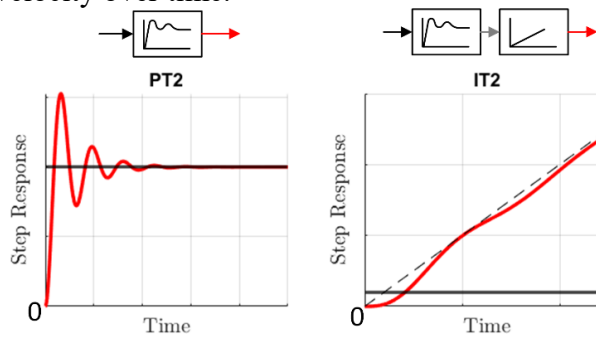

IT2

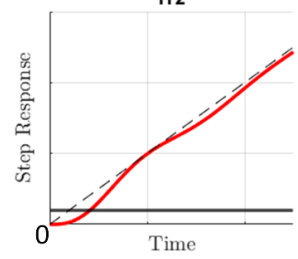

Figure 9: PT2 and IT1 step response (qualitative).

However, if an internal process signal is selected as the control variable, e.g. here the (load) pressure as the output variable, dynamic elements (those ones containing at least one integrator) of the model lie in a feedback path and act in the opposite direction to the direct forward direction between the input signal (control voltage at the valve) and the output signal (here now pressure or driving force). Such branches inevitably generate zeros in the transfer function $G_{y}(\mathrm{~s})$, see figure (10) (here again, for simplicity and clarity, the idealized LTI model is used). These zeros, in turn, can have unusual and usually unpleasant effects on the transmission behaviour if they are located near pole points and/or near the imaginary axis of the $s$-plane. For example, if a zero point is closer to the origin than the nearest pole, the system will show overshoots in the step response, even if the poles do not allow any oscillations at all, see figure (11).

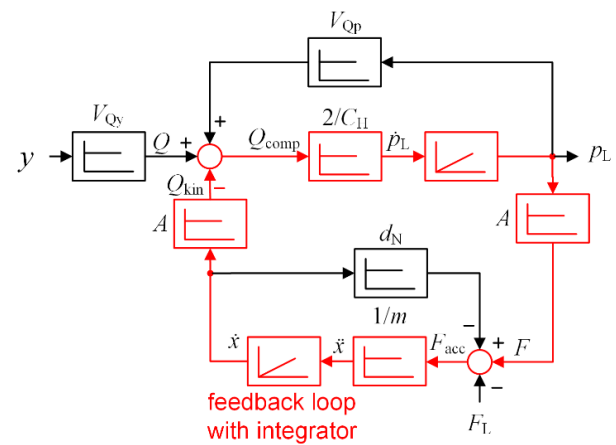

Figure 10: Idealized block diagram with load pressure as control variable.

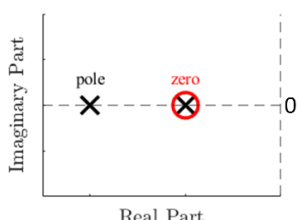

Real Part
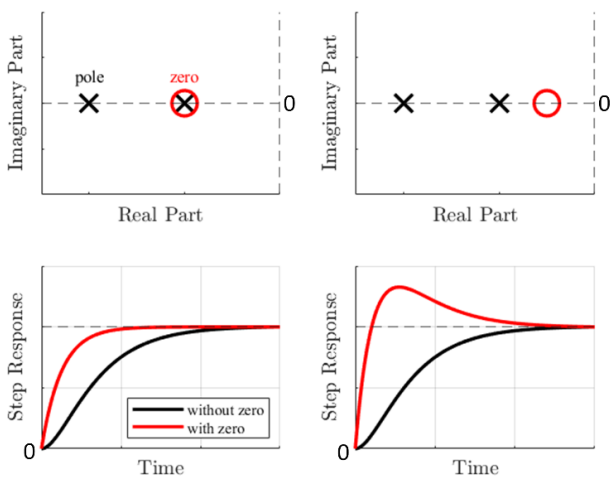

Figure 11: Characteristic pole/zero constellations (qualitative).

In many cases, the load force is not an external force (not an input signal), which is given from outside independently of the system, as is the case with a lifting movement, for example. In forming processes, the load force depends decisively on the motion state of the forming piston, i.e. specifically on its position and speed. However, these relationships are often rarely known in such detail that they can be described mathematically unambiguously and used meaningfully in a model, see Figure (12). In such cases this fact has far-reaching consequences for the controller design and is often the background to many unsatisfactory solutions. 


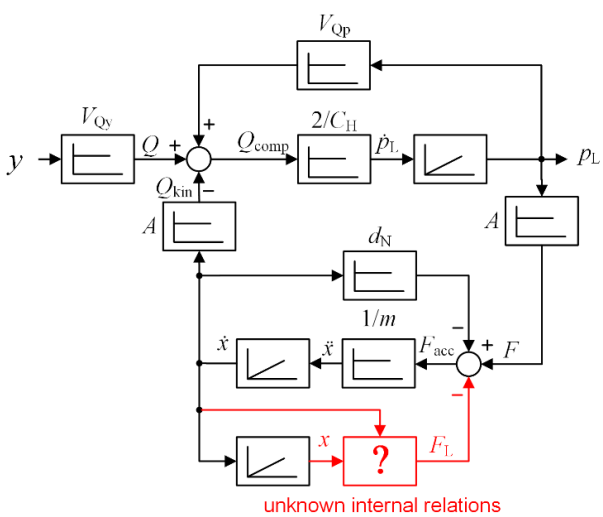

Figure 12: Idealized block diagram with unknown internal relations.

\section{CLASSIC CONTROL CONCEPTS FOR SERVO DRIVES}

\subsection{Basic Rules}

Controlled hydraulic drives are often said to be complicated in terms of control technology, the control concepts are very diverse and casespecific, and there is no transparent systematic approach. However, if one takes a closer look at the subject, one must contradict this assertion, at least in this drastic form.

Of course, the situation with electric drives is comparatively simple. Electrical positioning axes are usually controlled by a three-loop cascade controller, sometimes also by a three-loop (state space) controller [3]. Here it is assumed that they have sufficiently high-dynamic output stages, so that there are almost recipe-like instructions for the controller design that lead to fixed design formulas. In contrast to the hydraulic case, electric drives as controlled systems are by nature mostly over-damped ( $D>>1)$, which simplifies the control task, since it is easier to reduce the damping of an over-damped system than to calm a system that is susceptible to oscillations. Perhaps it can be said that the basic conditions of the hydraulic case (parameter dependency, actuator dynamics etc.) are somewhat more difficult than in the electrical case. Nevertheless, control structures and control parameters also result quite systematically for hydraulics if some elementary basic rules of control engineering are observed. Non-compliance with these basic rules is often the cause of the difficulties mentioned. Such basic rules are [8]:

1) The actuator (= control valve) should be as fast as possible, otherwise its dynamics must be considered when designing the controller.

2) If the controlled system itself has an integral behaviour, the controller must not have any pure integral parts.

3) Integral controller components serve on the one hand to correct disturbance variables and on the other hand to provide stationary control signal components, so that these have not to be generated permanently via a remaining control error or the proportional control component respectively.

4) Integral controller components generally have a destabilizing effect because they reduce the phase margin in the frequency response.

5) Stationary control signal components should be generated by appropriate offset or feedforward instead of integral feedback controller components. In this way, the operating point is adopted more quickly via the feedforward signal components without having the destabilizing effects of a closed feedback circuit. However, this compensatory measure requires more precise knowledge of the structure and parameters of the controlled system in the form of an appropriate mathematical model.

6) Zeros in the transfer function are caused by dynamic components in the feedback or by branches parallel to the forward branch of the controlled system. Depending on the position of the zeros, they can have a strong influence on the dynamic behavior, which is why they should be compensated by an upstream filter element. This compensation, which may depend on the operating point, requires knowledge of the structure and parameters of the mathematical model of the controlled system.

7) State controllers of higher than third order are usually problematic, since the generation of the additional state variables necessary via measurement, observer models or (possibly adaptive) differentiation algorithms is usually not easy.

8) The three-loop control is a special version of the state control for positioning drives with a sufficiently highly dynamic actuator. The 
advantage is that you can assign a defined effect to the inner return loops directly (and not only via the pole positions).

9) The control signal limitations that for energy reasons always do exist limit the possibility of correcting the eigendynamics of the system by means of controls. Multi-loop controls are particularly vulnerable here. Approaches to convert these controller structures into singleloop pole compensation algorithms can be helpful here [8].

\subsection{Case Studies}

\section{Position control of the hydraulic cylinder drive}

The situation for the position control of a hydraulic cylinder drive is as follows: Because of the free integrator at the end of the controlled system, this has an integrating behavior in the position control and there is no fundamental control error in the reference behavior with a simple $\mathrm{P}$-controller $\left(y=K_{\mathrm{P}} \cdot e\right)$, provided that static friction is negligible.

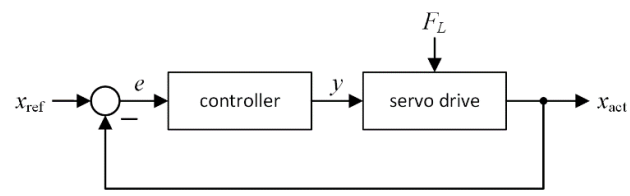

Figure 13: Single-loop position control.

However, a permanent control error $e_{\text {stat }}$ follows under load or during movement, namely in proportion to the respective cause load force and/or speed.

$$
e_{\text {stat }}=\left(\frac{V_{Q p}}{V_{Q y} A} \cdot \frac{1}{K_{p}}\right) \cdot F_{L}+\left(\frac{A}{V_{Q y}} \cdot \frac{1}{K_{p}}\right) \cdot \dot{x}
$$

However, with the controller coefficient $K_{\mathrm{P}}$ as the only degree of freedom of the P-controller, at best the requirement for a sufficiently safe stability of the controlled positioning drive can be met. For further requirements, controllers with more degrees of freedom (poles and zeros) are required.

If the actuator is sufficiently fast, the three-loop controller with speed and acceleration feedback is ideal. In particular, the tendency to oscillate can be relatively well suppressed here via acceleration feedback.

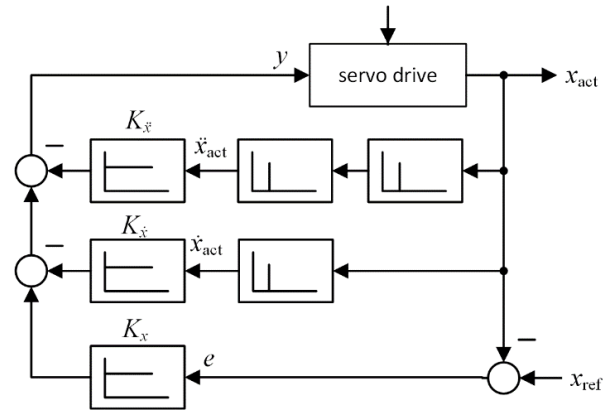

Figure 14: Three-loop position control.

A significant increase of the eigendynamics finds its limits quite quickly in the control signal limitation, but such an increase is not a primary goal for the mostly quite highly dynamic hydraulic drives. However, this control with additional speed and acceleration signal requires the availability of the necessary auxiliary control variables. Here, the speed can usually be determined by differentiation of the position signal, but for acceleration this procedure is much more difficult, due to several A/D quantization effects. Alternatively, a fading feedback loop with the difference pressure is possible. This is a direct equivalent to current feedback in the cascade control of electric drives. One way to completely bypass this procurement of the two auxiliary control variables is to implement this variant of pole assignment control by means of an extended single-loop compensation controller. Ideally, however, this requires real time computers that can handle floating point operations and a somewhat more sophisticated implementation of the control algorithm.

Even with the three-loop controller, there is a remaining permanent control error in the disturbance behavior. Pure integral controllers are not possible for reasons of stability. A PIcontroller as a way out creates a parallel branch and thus as mentioned above a system-related zero. This zero is even dominant and creates a characteristic overshoot. These can be avoided by a switching integrator (i.e. type of anti-windup integrator), which only becomes active within a relatively narrow band around the setpoint. 


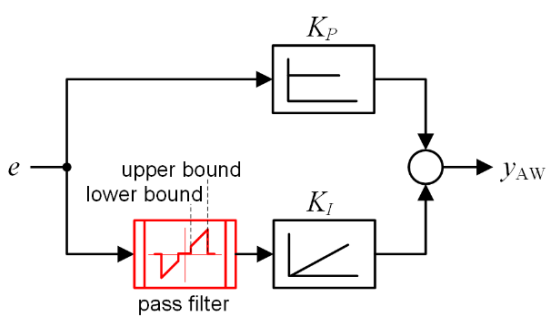

Figure 15: Anti-windup PI-controller structure.

Alternatively, the zero can be compensated by an upstream filter element. To dimension this compensating element, however, the system model must again be well known with high parameter accuracy.
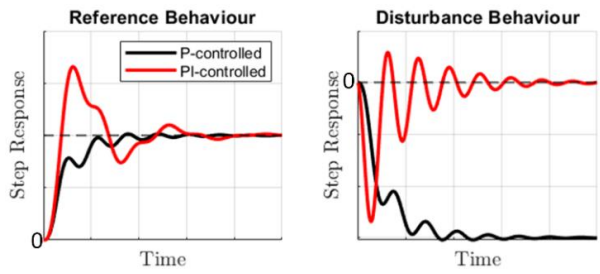

Figure 16: P- and PI-controlled step response (qualitative).
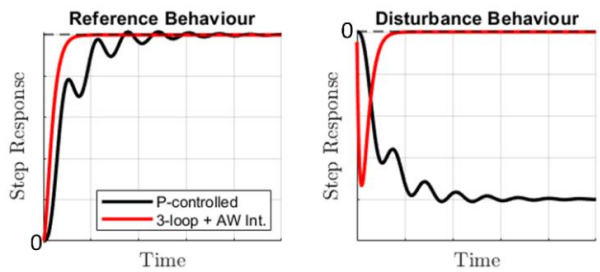

Figure 17: P-controlled and three-loop + anti-windup controlled step response (qualitative).

If actuator (= valve) dynamics cannot be neglected in relation to the main system dynamics, a frequency response based controller design using the bode diagram of the open loop system is recommended. This is somewhat more complex to carry out than the other methods mentioned so far, but can be applied practically without any restrictions in regard to the controlled system. With the design strategy often referred to as PT2 analogy according to Lunze [4], a purposeful and relatively design-safe design process based on the bode plot is available, that can theoretically be applied to any transfer function, even with dead time. With the appropriate use and combination of basic LTIelements (P- and I-elements) and amplitude and phase shaping elements (lead-lag-compensators or PDT1- and PPT1-elements respectively) the following design criteria should be met:

1) Provide an integrator if the system itself has none.

2) Phase margin of about $70^{\circ}$.

3) High crossing frequency (frequency at which magnitude crosses $0 \mathrm{~dB}$ line).

4) Magnitude slope of about $-30 \mathrm{~dB} / \mathrm{dec}$ at crossing frequency.

5) High magnitude attenuation for frequencies $>>$ crossing frequency.

In principle, this leads to single-loop controls and does not require the reconstruction of auxiliary variables, but can lead to control algorithms of quite high order if system dynamics are changed too far. However, such changes are restricted quite narrow due to the control signal limitations anyway. State space control using pole placement is a much discussed and often propagated highend solution. The three-loop controller just mentioned is (for positioning drives with a sufficiently fast actuator) nothing else than a special case of this state space control using pole placement. A big advantage of this controller is the existence of clear and easily algorithmizable design calculations. However, a general application of the state space control via pole placement often is not without problems. This concept requires the availability of all state variables, some of which, however, often cannot be measured or can only be reconstructed with a great deal of effort. The possibility of apparently being able to define "freely" the eigendynamics of the controlled system often leads to the design of "overbred" and highly sensitive controllers, which then result in malfunction of the control circuit and even instability. Last but not least, with model-based controllers based on the TwoDegree-of-Freedom principle, which has emerged strongly in recent years, a powerful and extremely promising alternative to state space controllers is available [8], see next chapter.

\section{Speed control of the hydraulic cylinder drive}

The basic principles for the controller design mentioned above are generally valid and therefore also applicable to other control modi of the drive systems. In the case of speed control, the integrator at the plant output is missing. The concept presented below is therefore useful 
according to the basic rules mentioned. To improve the transfer behaviour, an integral component is unproblematic here and should be used for load and disturbance compensation. However, the required control signal component should not be generated mainly based on the control error by means of this integrator, but via a forward branch/offset using the reference signal and the inverse stationary model equations (discussed in more detail in the next chapter). A possible overall configuration is shown in Figure 18

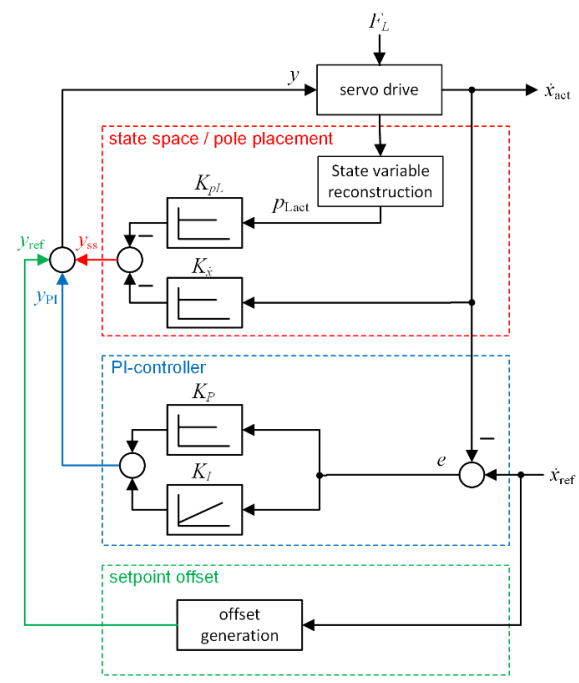

Figure 18: Structure of velocity control using a PIcontroller, a state space controller and a velocity offset in parallel.

\section{Pressure control of the hydraulic cylinder drive}

The basic problem with pressure control is that the compression volume flow required for the desired pressure profile is usually orders of magnitude smaller than the kinematic volume flow required for superimposed piston movement. Since any kind of volume flow can only be generated in the controlled drive via the control deviation, this situation leads to dramatic deviations of the actual pressure from the target pressure in case of superimposed piston movement. As already mentioned in the chapter about modeling, the causal relationship between load force and piston speed/position cannot be described in most cases in simple and transparent mathematical way. As a result, a reliable determination and thus compensation of the disturbance volume flow resulting from this piston movement is analytically impossible. However, the magnitude of this disturbance volume flow can be determined and calculated indirectly via the piston speed, which means that compensation of the disturbance volume flow is now possible by means of a corresponding control signal offset. With a sufficiently fast valve as the actuator element, this special form of feedforward control provides sufficiently good compensation of the movement, so that from a control engineering point of view, only one PT1 element effectively remains as the system to be controlled, see block diagram Figure 19. Due to the variable piston position (the variable hydraulic capacity of the chamber respectively), the time constant of the PT1 element is variable.

However, since this position-dependent time constant can thus be determined via the position sensor, an adaptive PI algorithm can be easily implemented here. This concept is ideally suited, for example, for high-precision pressure control of die cushions.
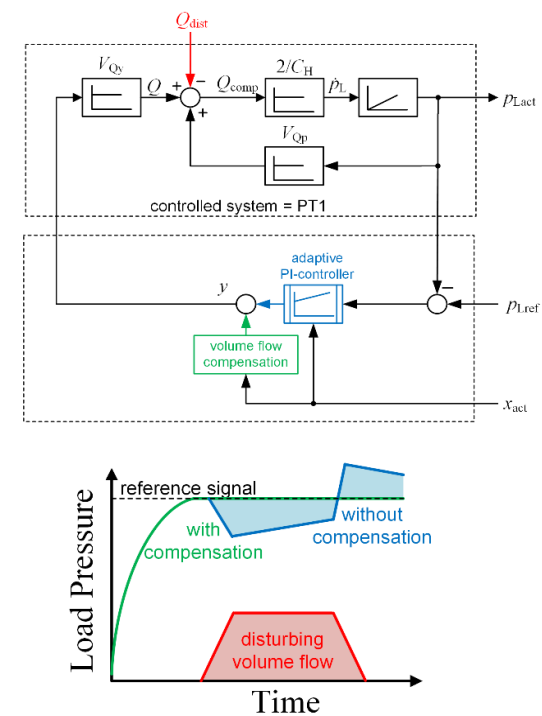

Figure 19: Structure of pressure control using an adaptive PI-controlled and qualitative control results.

\section{Chapter summary}

Using the example of the valve-controlled hydraulic cylinder drive, these examples show that control design for these drives is a transparent task that can be solved relatively 
purposefully, provided that a corresponding accurate model is available. With this knowledge, an adaptation of the controller to changed operating parameters and thus a turn away from a worst-case parametrization is also quite feasible. In principle, this also applies to fundamentally different drive concepts, such as pump-controlled drives.

These considerations show that the controllability of the systems goes hand in hand with the availability of structurally correct and correctly parameterized system models. If these models can be generated computer-aided with reasonable effort and with sufficient accuracy, the modelbased control based on the Two-Degree-ofFreedom principle is a modern method that will complete or replace the classic approaches in many areas, especially when higher dynamics are demanded. This concept will be briefly discussed in the next chapter.

\section{MODEL-BASED CONCEPTS}

\subsection{Model-based control according to the Two-Degree-of-Freedom concept}

Developments that have been unstoppably on the rise in recent years and that will replace or supplement the classic control concepts in many areas are model-based control concepts. At its core, this is a more sophisticated and systematic variant of the well-known pilot control. On the control theory side, it was motivated and scientifically proven by the theory of so-called dynamic flat systems. The simple basic idea of these concepts is that it does not matter where the correct control signal required for the desired course of the controlled variable comes from, the system to be controlled does not notice its origin anyway. The control signal has only to be correct in the sense that it meets the control requirements. This control signal is generated in classic control loops based on the control error. The term control error already makes it clear that one inevitably accepts that the system is at least temporarily not at the operating point that is specified as the current target via the command signal. If you want to keep this error small, the controller, as the coupling element between the control error and the control signal, must be set to "armed", i.e. the loop gain must be driven up. Therefore, the stability limit is increasingly being approached. In order to avoid the impending instability, higher-quality controllers (e.g. pole placement using state space controllers) are used that strongly intervene in the system's own dynamic. With increasing complexity, these higher-quality controllers are of course becoming more and more susceptible to signal errors or parameter fluctuations.

This is where the model-based concepts come into play. On the one hand, today one has (or should have) very precise knowledge of the real system behaviour. On the other hand, also excellent mathematical computing tools that allow such models to be calculated and evaluated even in real time are available. In the modelbased method, target trajectories are generated in a so-called trajectory generator, from which, based on a mathematical model of the system, a feedforward pilot controller calculates control signals that "impress" the desired output trajectories as precisely as possible.

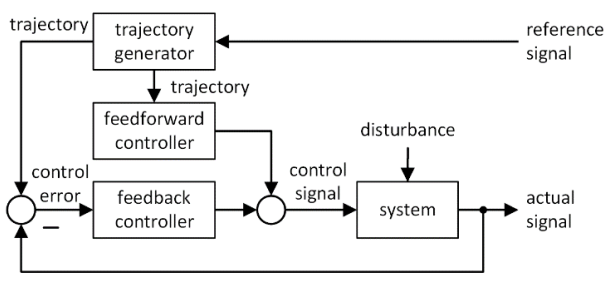

Figure 20: Basic structure of the Two-Degree-ofFreedom control concept.

If the system is to be guided at least in part via this input based control branch, only such trajectories may be generated which can be realized by the physical drive system: limits and operating point-dependent power restrictions must therefore be considered when specifying sensible target trajectories. Therefore, step signals as a command signal are of course pointless, or they must be smoothed out sensibly by the trajectory generator. If this requirement is met, the problem of signal limitations that is always present in conventional controller design processes, is avoided or at least largely mitigated. The design goal is achieved when the control signal profile provided by the feedforward pilot control branch produces the desired target curve in good approximation even without feedback control. With this structure, the feedback 
controller only has the task of correcting the effects of inaccuracies of the model used in the pilot control system, as well as supressing the remaining influence of disturbance signals. Without disturbance signals and with an ideally exact model, the control signal based on the model-based feedforward branch would force the desired trajectory. The feedback controller would recognize no control deviation and would not intervene.

Some big advantages of this approach are:

- Nonlinearities have no or only a minor effect, provided that they are considered sufficiently precisely in the model used in the feedforward pilot branch.

- Parameter fluctuations do not have a major impact.

- A comparatively simple feedback control structure can be selected, and the feedback control parameters can be weighted moderately.

- The problem of stability is reduced, only feedback components of the control signal can cause instability.

In order to be able to use this approach for the position control of valve-controlled servo drives e.g. effectively, the following basic conditions must be met (see [5], [6] for further details e.g.):

- A target trajectory that can be differentiated three times must be generated.

- The chamber pressures $p_{\mathrm{A}}$ and $p_{\mathrm{B}}$ must be measured.

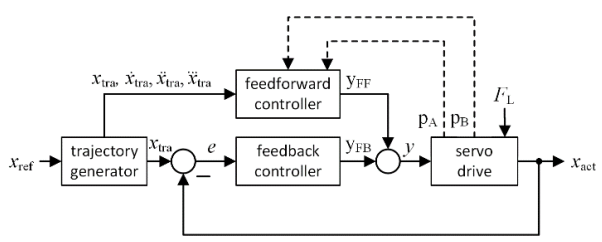

Figure 21: The Two-Degree-of-Freedom concept applied to the position-controlled servo drive.

As Ströbel has shown [5], implementing this idea in accordance with the control of dynamically flat systems in the case of the hydraulic cylinder drive is not without problems. On the one hand, the real physical dependencies can often hardly be described exactly and reliably using a parametric (=formula-based) mathematical model.
Examples are the real flow conditions over the control edges as well as the influences of friction and leakage. On the other hand, the necessary case distinctions when describing the flow behaviour via the control edges of the control valve make the creation of a real time capable algorithm considerably more difficult. Although Ströbel was able to create a suitable algorithm, he also had to recognize that the resulting complexity of a generally applicable approach would significantly hinder the widespread dissemination of this theoretically good concept.

\subsection{Practical alternative using a characteristic map}

So in [5] a slightly different, more pragmatic approach is taken. The basis for this was the following principle consideration: Ideally, the pilot control branch should determine an actuating signal that generates the entire volume flow required, i.e. both the kinematic volume flow for moving the piston and the compression volume flow for building up pressure in the cylinder chambers. On the other hand, when the system state changes, the compression volume flow is usually orders of magnitude smaller than the kinematic volume flow. There is also no convincing reason why the pilot control branch must generate the entire volume flow required, as the feedback controller did previously. It would already relieve the feedback controller dramatically, and thus allow a much weaker and therefore more stable controller parameterization if the pilot branch could at least generate the signal component for the kinematic volume flow.

The entirety of the operating points belonging to purely stationary states of motion (or kinematic volume flows respectively) is exactly represented by the characteristic map of the drive. The creation of the same was based on the boundary condition that the current pressure changes and thus the compression volume flows were zero, or in other words: compared to a general dynamic movement, the respective compression volume flow is generally missing in the characteristic map. Conversely, this fact means that significant parts of the control signal can be determined from the characteristic map of the drive if this is known, that is to say if this is somehow available by calculation or measurement technology. The 
characteristic map represents the stationary solution of the corresponding mathematical model equations of the drive and can therefore be derived from construction plans and catalogue data of the drive components mostly in good approximation. On the other hand, it can in principle also be determined by measurement from the data triplet $\left(F_{\mathrm{L}}, y, \dot{x}\right)$ or $\left(p_{\mathrm{L}}, u, Q\right)$ respectively: each operating point that is taken up when passing through a trajectory represents such a data triplet.

If the map is in a form in which the control signal $y$ of interest is the output variable, the important -because dominant!- stationary control signal component for the displacement volume flow can be interpolated in real time depending on the operating point and used to control the valve. Since only stationary components can be generated in the pilot control branch by means of the characteristic map, a trajectory that can be differentiated once is sufficient to determine the control signal $y$, whereby a smoother trajectory of higher order still remains meaningful in order not to demand impossible accelerations. The gradient of this trajectory directly corresponds to the variable stationary speeds or the kinematic volume flow to be realized and, from the feedforward controller's point of view, is thus the reference variable. The load force $F_{\mathrm{L}}$ or the load pressure $p_{\mathrm{L}}$ as the disturbance variable is reconstructed online based on the two chamber pressures.

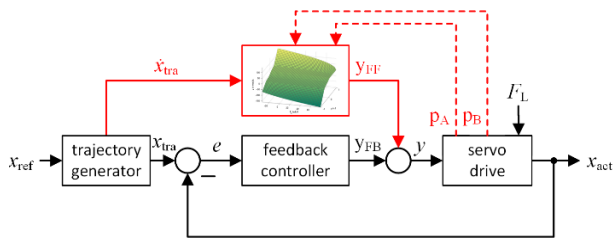

Figure 22: Characteristic Map based implementation of the Two-Degree-of-Freedom concept applied to the position-controlled servo drive.

This concept was tested on valve-controlled hydraulic cylinder drives and on die cushion presses. Together with a subordinate classic feedback controller, this concept according to the Two-Degree-of-Freedom concept delivered excellent results, see [5] and [7] for example. The concept in this form is of course also transferable to other drive variants and offers a number of additional expansion options.

With this map-based approach, the term TwoDegrees-of-Freedom can be also assigned a clearly specified meaning:

- Feedforward part (model-based): takes care of the most exact possible implementation of the stationary process components, which are traditionally realized by a high loop gain. For this, the most exact possible representation of the stationary transfer behavior, i.e. the characteristic map, is necessary.

- Feedback part (physical state based): takes care of the non-considered dynamic process components. Should realize a dynamically acceptable, i.e. above all a well damped (stable and robust), dynamic transfer behavior by constant feedback monitoring and correction. The better the feedforward part, the smaller the corrective interventions.

The two parts can thus be meaningfully and independently dimensioned according to their respective areas of responsibility.

\subsection{Characteristic map optimization}

Whether as the basis for a conventional controller or as the basis for a model-based control based on the Two-Degrees-of-Freedom principle, the availability of more suitable, i.e. sufficiently accurate and easy-to-use mathematical models is a basic prerequisite for realizing highly precise dynamic drive controls. Of course, the question immediately arises as to how these models are determined in terms of structure and parameters. It is very common to define model equations that appear sensible on the basis of physical considerations and to first set all unknown or uncertain parameters here as variables. The specific values of the present case are then determined experimentally, for which there are a number of different identification methods. The equations parameterized in this way form the mathematical model of the system and then e.g. the basis for the controller design.

With the Two-Degrees-of-Freedom principle according to the theory of dynamically flat 
systems, the control signal for desired trajectories is determined analytically (= formula-based) at the current operating point by evaluating these in general non-linear system equations. A certain weakness of this method is that the analytical model equations are structurally fixed, based on physical considerations and assumptions, and only their parameters can be experimentally determined or optimized in the sense of a curve fitting. Structural errors in the model cannot be corrected. In the case of the hydraulic cylinder drive, however, it is sufficient and sensible to determine the dominant control signal component for the displacement volume flows from the solution for stationary conditions. However, the entirety of all stationary solutions is nothing else than the characteristic map of the drive in question. Mathematically, this map forms a nonlinear surface $Y$ of a two-dimensional input space with input quantities $X_{1}$ and $X_{2}$.

$$
Y=f_{n l}\left(X_{1}, X_{2}\right)
$$

Or in the specific case of the cylinder drive:

$$
\dot{x}=f_{n l}\left(F_{L}, y\right)
$$

Every point of this surface is a possible stationary operating point of the drive. Conversely, every real occurring and thus measurable stationary operating point represents a point of the real surface. The totality of all real occurring stationary operating points thus defines a point cluster which, if the model is correct, should lie exactly on this surface. Ströbel uses this fact for an extended optimization process of the map. First, based on the known or roughly estimated model structures and parameters, a first, usually only partially accurate, steady-state characteristic field is determined by solving the model equations and stored as a non-parametric (= number-based) white box model (index W) in the form of a two-dimensional lookup table (LUT), which mathematically represents the corresponding surface $\mathbf{C} \mathbf{M}_{\mathrm{W}}$.

$$
\mathbf{C M}_{W}: \dot{x}=\mathbf{L U T}\left(F_{L}, y\right)
$$

In a second step, the triples $\left(F_{L}, y, \dot{x}\right)$ that occur during regular operation are measured. Since these are generally "contaminated" due to the dynamic processes that are actually taking place, for example due to oscillations and measurement noise, they are first filtered by suitable measures and the stationary parts are extracted. As a result, there is a set of black box observations (index B) which represent $N$ stationary operating points.

$$
\mathbf{O P}_{B}=\left(\begin{array}{ccc}
F_{L(1)} & y_{(1)} & \dot{x}_{(1)} \\
\vdots & \vdots & \vdots \\
F_{L(N)} & y_{(N)} & \dot{x}_{(N)}
\end{array}\right)
$$

If the model is accurate, the operating points are exactly on the surface. If they are next to it, this is an indication of one or more possible model errors and in this case the surface should be corrected or optimized accordingly to bring the model and observation into line. For this correction, Ströbel developed a method based on a special variant of neural networks (radial basis function networks). Based on both the original white box model and the black box observations, a new optimized gray box model is synthesized in the form of another numerical lookup table.

$$
\mathbf{C M}_{G}: \dot{x}=\mathbf{L U T}\left(F_{L}, y\right)
$$

This method has proven to be extremely suitable and quickly converging in various tests, even if only a few (e.g. fewer than ten) reliable black box observations are available. See [5] and [7] for further theoretical and experimental details.

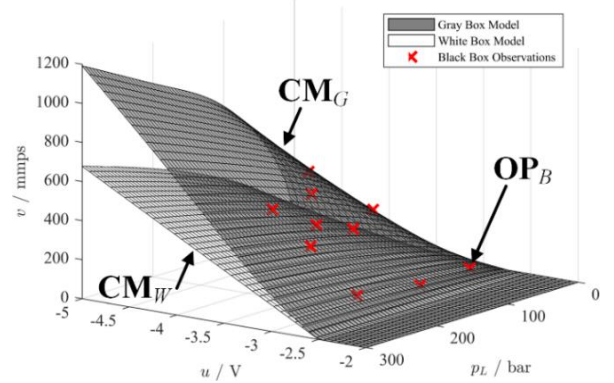

Figure 23: Illustrating the characteristic map optimization on the example of a die cushion press with positive overlapped valve control edges, taken from [7].

A major strength of this method is that the transition to a nonparametric model in the form of a lookup table, composed of many numerical data points, means that the model errors as a result of the mathematically rigid structure of the model equations can be easily compensated. In principle, each data point of the lookup table 
forms a degree of freedom to correct the model qualitatively and quantitatively, regardless of whether these errors are due to a faulty model structure or faulty model parameters. Another advantage is that no additional sensors are required for this correction, position and pressure sensors are sufficient. Since this process can run online in the background of the operation of the system, changes in the model parameters due to wear or aging can also be detected. In terms of condition monitoring, this can provide important information about the current state of the drive and/or about possible impending faults or failures.

\section{ENERGY ASPECTS}

\subsection{Concepts for continuous setting of hydraulic power}

When evaluating drive concepts, energy efficiency is becoming more and more important. The energetic consideration of drives can be divided into two parts: One question is which losses occur when power is transferred from the hydraulic power source to the consumer and then converted into mechanic power or motion within the motor. The other question is whether, and if so to what extent, power can be fed back and made usable when the load drives the motor thus works in the generating $2^{\text {nd }}$ or $4^{\text {th }}$ quadrant. As far as the first question is concerned, as is generally known and apart from special solutions such as the hydro transformer etc., hydraulics knows three well-known methods of power generation [1], [2]. The most important features of which are briefly listed in Figure 24.

\subsection{Resistance control as an energetic problem case}

As mentioned above, the resistance control is principally lossy. Nevertheless, it is interesting to compare the principal volume flow path of the valve-controlled servo drive with the electric circuit of the electric drive.

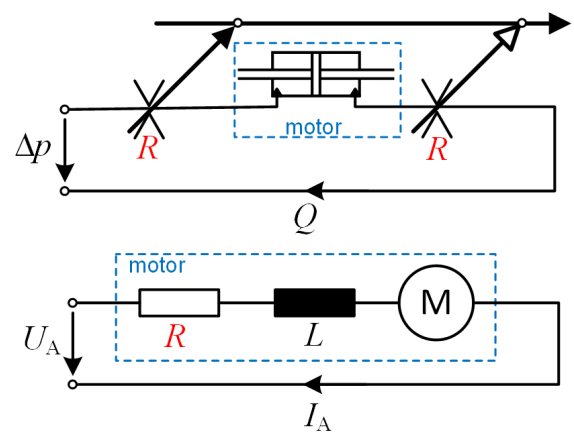

Figure 25: Principal comparison of the hydraulic and the electromechanical case.

Here a structural similarity is noticeable, but it is deceptive: In the electrical case of the circuit as well as in the hydraulic case resistors are in series with the force-generating element. But while in the hydraulic case the resistors are part of the actuator for power adjustment, in the electrical case they are part of the operating principle of the

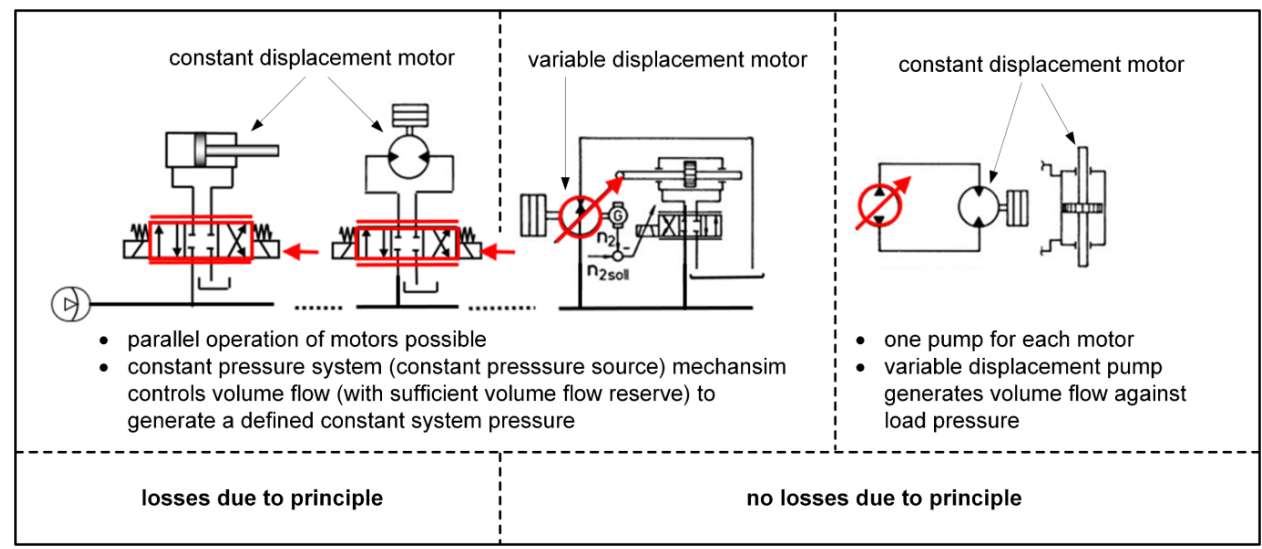

Figure 24: Practically relevant variants of the continuous hydraulic power setting. 
motor. However, a real correspondence would be given there, if an adjustable ohmic resistor for power setting at the consumer (=motor) would be introduced into the electric circuit. This variant would be physically possible here too, but in practice it is de facto meaningless for various good reasons (power loss, unfavourable characteristic map, etc.). On the other hand, both drive variants take their power from a central supply unit, a constant pressure network (hydraulic) or a constant voltage intermediate circuit (electric). Parallel operation of several consumers at these power sources is only possible by adjusting the power flow in each consumer branch to the required power. The question remains as to why this is possible without any losses in the electric case, but not possible in the hydraulic case.

The torque-generating motor current is controlled for the electric motor via the drive or armature voltage $(\mathrm{A}=$ armature, $\mathrm{M}=$ motor, $\mathrm{EMF}=$ back electromotive force) [1].

$U_{A}=U_{M}+U_{E M F}$

Whereas in hydraulics the power adjustment is achieved by reducing the supply pressure via pressure losses in resistors, the electrical drive technology has the advantage that with the inductance $\mathrm{L}$ of the coil in the current circuit there is an inertia-generating element which offers the possibility of generating the "correct" torquegenerating motor current via the time average value of the current flowing between the switching states $U_{\mathrm{A}}=U_{\mathrm{MAX}}$ and $U_{\mathrm{A}}=0$. At $U_{\mathrm{A}}=U_{\mathrm{MAX}}$ the resistance in the "electronic switching valves" (transistors, thyristors, IGBTs etc.) and therefore the power loss is minimal and at $U_{\mathrm{A}}=0$ no current flows and the power loss is therefore zero. Due to the inductance of the coil as an inertia creator, the current "trembles" at a sufficiently high switching frequency $f_{\mathrm{S}}$ (usually several $\mathrm{kHz}$ ), with a superimposed ripple, sufficiently smoothly around the value required to generate the torque.

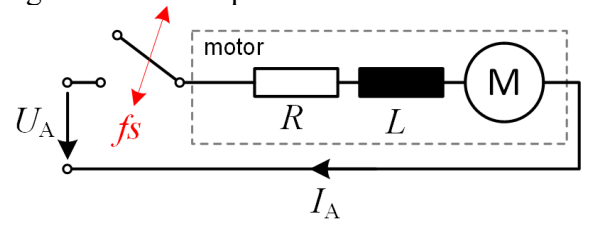

Figure 26: On-off switching of the electrical circuit.
In the output stage as a control element, therefore, no drive power is consumed, "only" the switching losses in the semiconductor components occur, whereby at high power levels and high frequencies the power losses can become so big that the corresponding components must be cooled with water and the energy advantage is at least partially lost again. This possibility of an at least in principle lossless voltage adjustment is a great advantage of electrical drive technology. In addition, even higher voltages than the existing supply voltage can be generated by special circuitry if required. (step-up converter). Unfortunately, there is no usable equivalent for this at hydraulics, because at electric case (= electron current) the flowing medium is practically massless and thus inertia-free but can be made inertial by inductors (e.g. coils). And the opening and closing of contacts is done by electric fields and not by movement of masses with corresponding impulse and thus permanently destructive forces due to the closing procedure.

This leaves the second question about the possibilities of energy recovery. This is unproblematic in the case of the electric drive, since the drive voltage $U_{\mathrm{M}}=U_{\mathrm{A}}-U_{\mathrm{EMF}}$ because of

$$
U_{E M F}=c \phi \cdot \Omega
$$

becomes negative at sufficiently high negative speeds $\Omega$ and thus feeds an electric current back to the power supply. Hydraulically, the power is generated in hydrostatic displacement units and these can in principle always also work as generators, so that one could think that power recovery (= recuperation) is possible with all three principles of hydraulic power setting mentioned. Unfortunately, this does not apply to the valve-controlled drive, and this is ultimately due to the rigid coupling of resistors, which are firmly attached to the slider as control edges and which prevent loss-free filling of the chamber on the inflow side when pulling forces. The fluid flowing into this chamber is then also fed by the pressure line and must be throttled via the control edges as throttle resistors, whereby the phenomenon of sucking already mentioned above can quickly occur. This critical condition can again be avoided using check valves. This solution to the suction problem is on the other 
hand essentially a workaround of the fixed control edge configuration of conventional control valves. As the chamber pressure diagram shows, position control is not unproblematic with pulling loads because of the kink in the characteristic curve at the critical load pressure $p_{\mathrm{L}}=-\alpha^{3} \cdot p_{0}$ [1], [2]. However, the complete problem with all the case distinctions for the various operating situations becomes obsolete if the asymmetry caused by the unequal piston areas is compensated by a corresponding reciprocal asymmetry in the flow rate coefficients in the control valve [2]. However, the associated turning away from the use of classic $4 / 3$ control valves does not necessarily mean to create a larger unwished variety of individual valve configurations that is hardly manageable in economic terms.

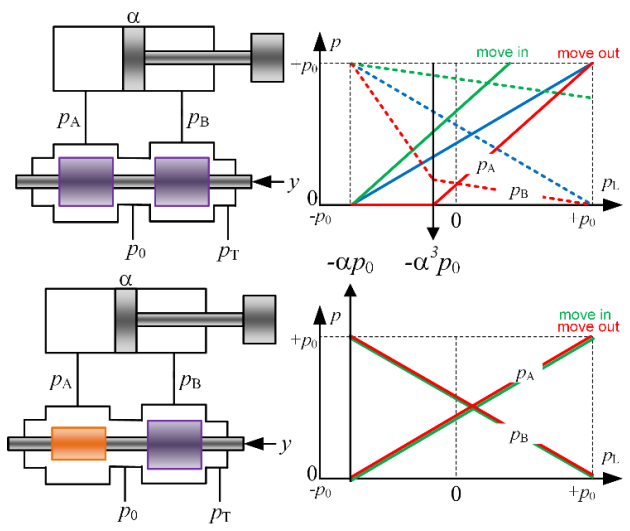

Figure 27: Symmetric and asymmetric valve control edges and corresponding qualitative pressure diagrams. The asymmetric case can possibly and easily be realized via two independent $3 / 2$ proportional directional valves which are coupled electrically.

There are some more examples that conventional concepts with classical control valves may lead to useful but often suboptimal solutions. Possibilities of creating alternatives here by means of other valve designs are known but have not yet been pursued or have been pursued only half-heartedly. Such approaches under the heading of resolved control edges have already been investigated in various cases at universities [11], but despite numerous interesting perspectives, they have unfortunately not yet found any significant systematic implementation in practice except some quite special solutions. Even if the importance of valve-controlled servo drives in hydraulics might decline further in the future, efforts should still be made to fully exploit the existing potential of this technology, which is unrivalled for some applications.

As far as power recovery is concerned, even in cases where it is possible in principle, it only makes sense if the energy generated can be used reasonably. This raises the question of storage, whereby in the case of a hydraulic drive on the engine itself, hydraulic power is initially generated, which in turn can only be stored directly as pressure energy in hydro accumulators. Here the possibilities are quite limited, not least for reasons of profitability. A conversion into other forms of energy that are easier to store is a possible way out, but it must not be neglected that each conversion of the form of energy is associated with losses. Here, the pump-controlled drive in principle has a slightly better position if it is driven directly by an electric motor as done in the so-called compact drive. Here there is the possibility of feeding back energy into an electric intermediate circuit (if existing and accessible), which makes it possible to supply other parallel running electric drives completely or partially with the energy fed back. It can also be expected that the latest developments in electrical power engineering e.g. by new battery technologies will bring new possibilities for the efficient use of regenerated electrical energy.

In view of the fundamental problems in energy recovery mentioned above, the current focus should be on avoiding unnecessary energy losses in hydraulic power supply control. Here there are comparatively simple approaches which are relatively simple and nevertheless very efficient and which, fortunately, are increasingly being used in practice. One approach that is becoming increasingly important, especially against the background of the digitalisation and Industry 4.0 concerns power adjustment on the supply-side. In the case of constant pressure supply, the hydraulic unit provides the volume flow which is required by the consumer as a resultant compression or kinematic volume flow. The aim must be to supply this volume flow as precisely as possible and to keep the excess volume flow required to maintain pressure as small as possible. This can be achieved by determining the volume flow over the pressure control valve using the 
signals for pressure and control slider position available in modern digitised pressure control valves. This volume flow is the actual value in a control circuit, that is set up for the outflow to keep this at a reasonable small value. The whole thing becomes even more precise and thus energetically more efficient if the pump is given a sensible pilot control signal to provide the very volume flow which is nominally required currently as the sum of all volume flows of all connected consumers. Determining this target value should be no problem in a digitised and fully interconnected process environment. [2]
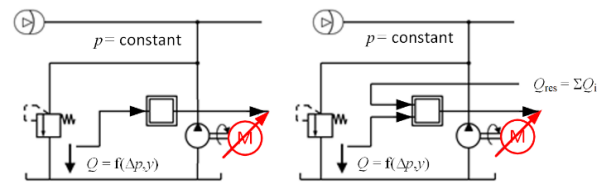

Figure 28: Energy saving solutions by smart hydraulic power supply.

\subsection{Pump control as an alternative}

According to what has been said above, two concepts with at least basically loss-free performance remain on the consumer side. However, since the realisation of a motor control is quite elaborate, especially if drives with differential cylinders are to be operated with it, only the pump control is left for the broad application. This umbrella term includes numerous variants, whereby the rotation of the pump drive shaft again can be generated by an electric motor [1], [2].

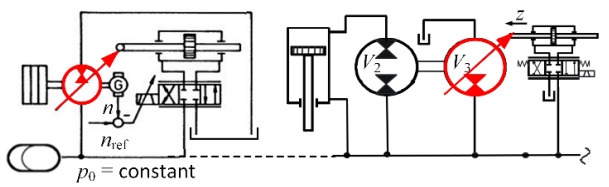

Figure 29: Rotatory vs. linear motor control (hydro transformer).

Figure 30 shows the basic principle of the pump control, which as one knows is basically suitable for four-quadrant operation. However, some things must not be overlooked in this very simple representation of the concept. When operating under a torque load, the fluid on the driving side is naturally compressed. If this compression volume were simply taken from the low-pressure side, negative pressures would be created there, and cavitation would threaten. At valvecontrolled drives, both chambers on consumer side are pre compressed to half system pressure.

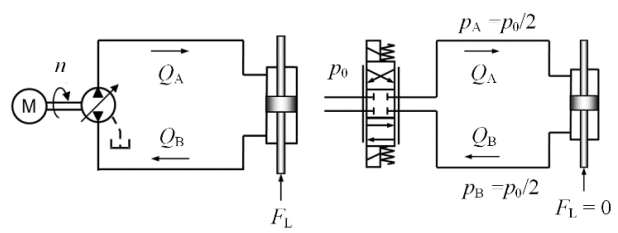

Figure 30: Comparison pump control vs. valve control.

In this case, an assembly with its own feed pump must be provided to generate the pressure on the low-pressure side. In addition to this unit for the load-dependent correct activation of the pressure on the low-pressure side, measures may be necessary, especially for drives that operate continuously with high loads, to feed the fluid stressed under load back into the tank for cooling and to replace it with fresh fluid from the tank [2].

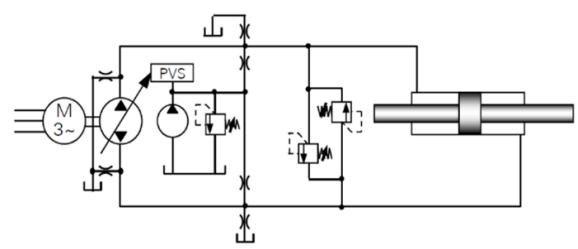

Figure 31: Closed circuit with preload pressure unit.

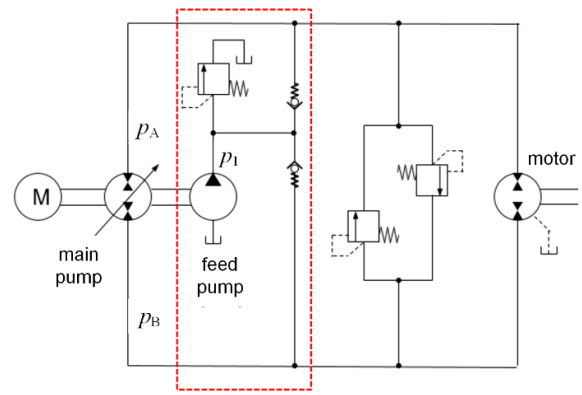

Figure 32: Closed circuit pump control with flushing and feeding unit.

Further circuitry measures are required if the motor is asymmetrical as in the case of a single rod cylinder. In this case the rod volume must be stored somewhere as a so-called pendulum volume depending on the stroke and fed back again during the return stroke. For this purpose, various more or less complex circuits have been developed, see Figure 33. 
a)
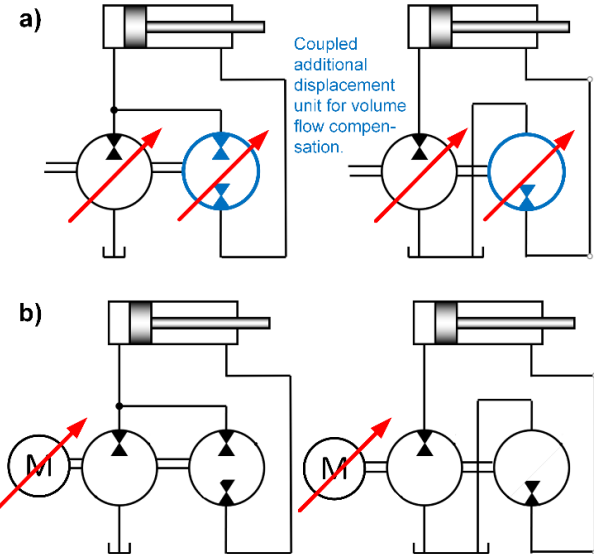

c)
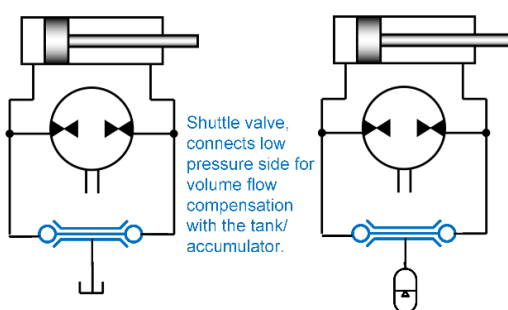

Figure 33: Differential cylinder drive with a) variable displacement pump control, b) variable speed motor directed pump control, c) shuttle valve

But a glance at the displacement units required in the case of a constant-speed electric motor a) clearly shows that these solutions were only used for very special applications for reasons of design and costs. However, when using constant displacement units and a variable-speed electric motor instead of displacement units b), the principle is basically the same but represents a more reasonable solution.
An even more simple and also cheap solution is to use a shuttle valve c). Here, however, there are certain switching effects when the low-pressure side is changed [1], [2], [12]. As an alternative to a tank, the pendulum volume can also be stored in an accumulator. Since the fluid volume to be stored is now stroke-dependent, the accumulator pressure increases with the stroke, starting with the withdrawn position. The actuator can be regarded as a plunger with a nonlinear hydraulic return spring. This effect must be considered when designing the controller. Figure 34 shows the dynamic model of such a pump-controlled cylinder drive. As can be seen, in comparison to the model of the valve-controlled cylinder drive, the servo valve has been replaced by a pump which generates a variable flow in both directions, either by varying the swivel angle at constant pump speed or, increasingly important, with a fixed displacement pump driven by a variable speed electric motor [1], [2], [12]. The model has only one motor chamber with an independent dynamic pressure build-up. If one defines the clamping pressure on the low pressure side with retracted piston and thus with minimum pendulum volume as the new zero pressure level, a structure for the cylinder results which differs from that of the valve-controlled drive only in two points: the term $1 / \mathrm{C}_{\mathrm{H}}$ instead of $2 / \mathrm{C}_{\mathrm{H}}$ and the stroke-dependent pressure in the driven chamber [1], [2], The halving of the proportionality factor expresses that now only one chamber (instead of two in case of valve-controlled actuators) is active as force generator. In cases with a sufficiently large accumulator and small pendulum volume, the stroke-dependent force effect of the low-pressure chamber (dashed force branch) can be neglected. Appropriate control concepts for this structure again result from implementing the basic rules presented above.

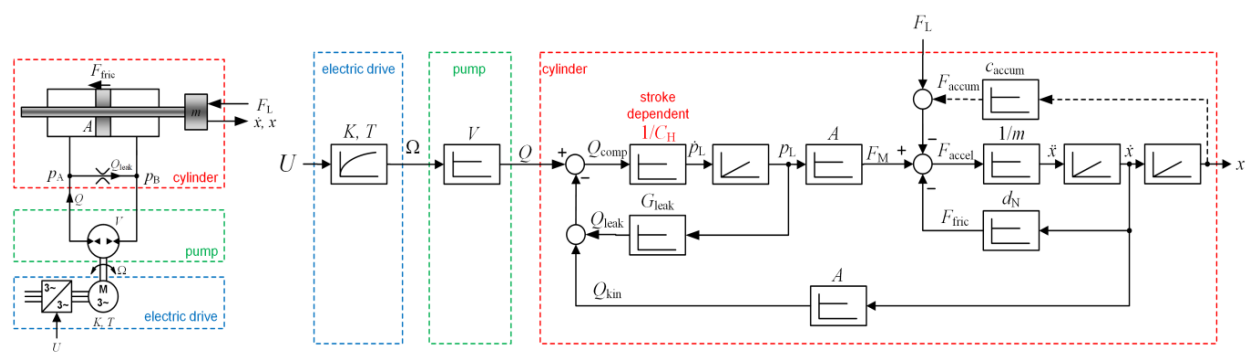

Figure 34: Pump-controlled system: simple scheme (left, switching valve and tank respectively accumulator for pendulum volume storage not drawn) and block diagram (right) 
In the specific selection of the fixed displacement pump for applications with variable speed drive, special attention must be paid to operating points where no or almost no volume flow is required, for example when reversing direction. However, many pumps require some drag fluid due to the relative movement of rotor and stator for internal lubrication. Here, tribological aspects also play an important role in the selection of materials. A consciously accepted larger leakage can help here but is questionable from an energy point of view. On the other hand, a leakage "smears" somewhat the reversing point with the change of the lowpressure side. These detailed questions are currently a R\&D-topic and there is certainly still potential for further developments.

\subsection{Electrohydraulic compact drives as an alternative to electromechanical linear axes}

With the pump-controlled cylinder drive with a variable-speed electric motor, a drive variant has been developed that is often referred to as a compact drive [12]. This attribute compact is perhaps less to be understood in the geometric sense, but rather expresses the fact that this is a standalone electro-hydraulic linear axis with the typical features that are otherwise only found in electromechanical drives. As an interface to the other process peripherals, it has an electrical power connector and a bus interface. The hydraulic transmission of the power generated in the electric motor can avoid numerous weaknesses of the electromechanical drives, where as you know, for example, the conversion of the rotary motor movement into a linear stroke movement via threaded spindles or similar converters is not unproblematic, especially with large strokes [12]. The possibility to store the pendulum volume in a hydraulic accumulator instead of in a tank has the advantage that the last component of the system, which requires a defined installation orientation, has been eliminated thereby. An electrohydraulic linear drive with an accumulator can be used in applications where the axis can swivel in all spatial directions. Even working in overhead positions is no problem any longer [9]. A disadvantage of the accumulator-afflicted version is that the pendulum volume to be stored increases with large strokes, which makes relatively large-volume hydraulic accumulators necessary. Here the multi-chamber cylinder as a special design variant can be a way out [9]

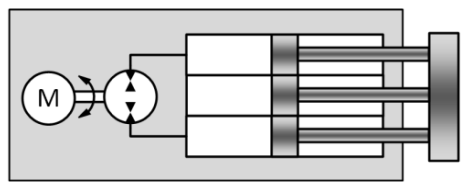

Figure 35: Simple scheme of a pump-controlled multichamber cylinder drive.

In this case five pressure chambers and one idle chamber are moved synchronously, whereby these chambers are connected in such a way that the drive has a resulting area ratio of $\alpha=1$ like a synchronous cylinder and still has only one piston rod. In addition, this variant allows a rapidmotion mode in both stroke directions, whereby switching between rapid and power mode is possible in any position [10].
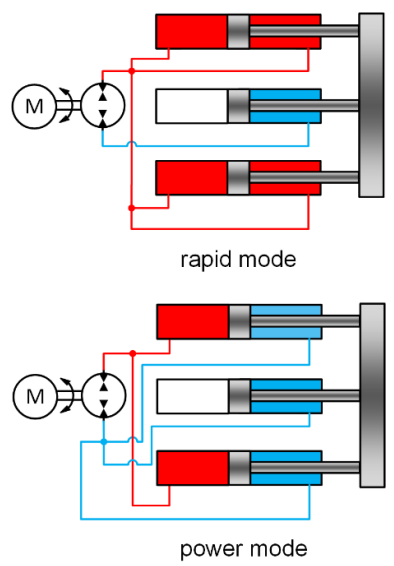

Figure 36: Multi-chamber cylinder drive connections for rapid mode and power mode.

Since there is no more pendulum volume, the hydraulic accumulator only needs to take up the compression volume and is correspondingly small, which in turn enables the realization of very long-stroke drives [9].

Of course, all these linear axes subsumed under compact drive do not normally have the flushing and refeeding unit which classic industrial pumpcontrolled drives do have. They are therefore 
probably more suitable as positioning actuators than as servo drives, although the limits and possible remedies have not yet been researched. In any case, the heat input is significantly lower than with valve-controlled drives. Since the drive principle is not based on controlling pressure losses at control edges, there is nothing any more to be said against solutions in the low-pressure range. This could be interesting for numerous applications which are electromechanically problematic for one of the reasons mentioned above, but which are above the range covered by standard pneumatics in terms of performance. A perhaps still somewhat visionary aspect of such a low-pressure variant today are current research activities, that investigate whether and, if so, which components of such a drive could be made entirely or partially of plastic, what would open alternative new production possibilities.

\subsection{The variable-speed electric drive as a component in hydraulic systems}

The remarks of the last chapter made clear that in the future the electric motor will play an increasingly important role in hydraulic drive technology. For this reason, a brief look now shall be included at some relevant aspects of this increasingly important component in the modular building kit of a hydraulic engineer. As a representative example of an electric motor, the classic externally excited direct current motor will be considered below. This motor may seem antiquated, especially in its brushed elementary version, but apart from the reluctance motor it has the advantage of obeying the same physical laws in a very transparent form as all other electric servo motors in use today, what makes understanding basic laws and dependencies easier. In addition, its stationary and dynamic behaviour is almost ideal, so that, to put it simply, other electric motor principles to be used as servo drives attempt to impose to these motors the stationary and dynamic behaviour of this DC machine e.g. by vector based motion control concepts realised by sophisticated electronic circuitries.

\section{Electrical DC drive basics}

In the DC machine, the creation of torque is based on the Lorentz force as a force effect on moving electric charge carriers, i.e. the electrons of the electric current flowing in the winding wires, which are permeated by a magnetic field with the flux density $B$. To drive this flux quantity, a armature voltage $U_{\mathrm{A}}$ is applied to the winding strands as a potential quantity [2].

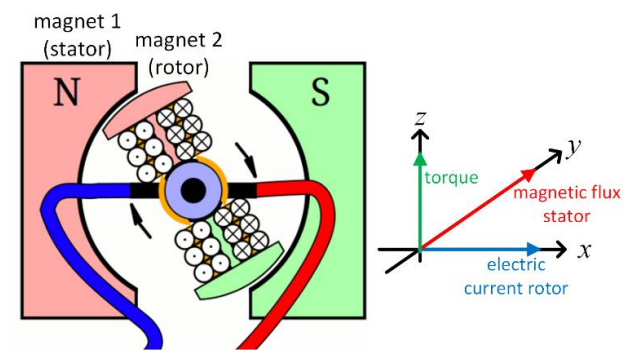

Figure 37: Operating principle of a DC motor.

However, as soon as the motor moves, this voltage $U_{\mathrm{A}}$ is reduced by the speed-proportional induction voltage $U_{\text {EMF. }}$. At least, it is precisely the differential voltage $\Delta U=U_{\mathrm{A}}-U_{\mathrm{EMF}}-U_{\mathrm{R}}$ driving the current $\mathrm{I}_{\mathrm{A}}$ in the windings and thereby generating the torque $\mathrm{M}_{\mathrm{M}}=\mathrm{I}_{\mathrm{A}} \cdot \mathrm{c}_{\Phi}$. Nevertheless $U_{\mathrm{A}}$ is the power - directing variable.

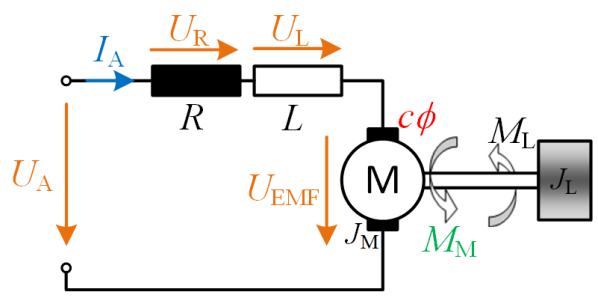

Figure 38: Scheme of a DC motor.

Based on the elementary equations for the Lorentz force on flowing currents in a magnetic field, for the voltage induction during the movement of charge carriers in a magnetic field and applying Newton's equation of motion, one obtains the well- known relationship as stationary model:

$\Omega\left(M_{L}, U_{A}\right)=\frac{1}{c \phi} \cdot U_{A}-\frac{R}{(c \phi)^{2}} \cdot M_{L}$

And again, under the assumption that the control element (output amplifier) is dynamically much faster than the drive itself, one obtains the dynamic model of the DC motor also quite simply but in contrast to the hydraulic case without the necessity of such questionable idealizing 
assumptions and without operating point dependent linearization. The corresponding block diagram is shown in Figure 40 [1], [2].

\section{Some selected comparisons between the electric and the hydraulic drive}

In this approximate view, the characteristic map of the DC motor according to equation (19) represents a linear plane and is therefore very simple compared to the (multiple) non-linear plane of the characteristic map of a real hydraulic cylinder drive.
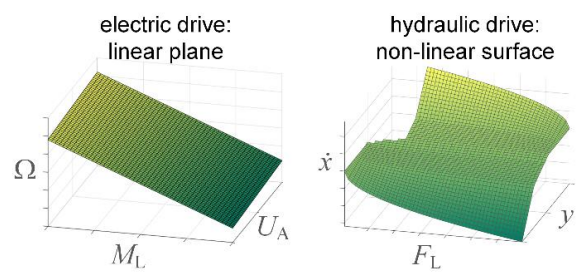

Figure 39: Principal comparison of the characteristic maps of electric and hydraulic drives.

As can be seen in the comparative block diagram in Figure 40, there is a structural similarity between the electric and the (idealised) hydraulic case of the dynamic model. However, despite this structural similarity, there are also a few significant differences:

- The parameters of the hydraulic case are partly strongly dependent on the operating point and are the result of linearization calculations, whereas the parameters of the electrical case are largely constant.

- Both models are second order systems that can in principle oscillate. However, with the parameters usually occurring in everyday practice, this oscillatory case is rather rare in the electrical case in contrast to the hydraulic case.
- In the electric case the force or torque generating variable is the current, i.e. a power flow variable, and in the hydraulic case the pressure, i.e. a power potential variable. However, since a potential variable can occur alone, but a flow variable requires a simultaneously occurring driving potential, the well-known fact results that to maintain a force in the hydraulic case, in principle, no power flow is required, but in the electrical case it is.

There is another difference between the hydraulic and the electric servo drive: with hydraulic drives as hydrostatic drives, the pressurized fluid is completely enclosed ("chambered"). During the movement of the chambers, there are relative movements between moving and still standing parts. At these contact points, leakage must be prevented by means of appropriate seals without unacceptably high frictional forces occurring. With increasing relative speed, however, the frictional forces do increase, and speed becomes a limiting factor.

With electrical drives, there are no contact points between bodies with relative movement, apart from the bearing of the rotor shaft. With electric drives, however, the force density in relation to a given available installation space is 5-7 times lower than in the hydraulic case and thus the force or torque here is the limiting factor.

Hydraulic drives generate power as a product of force and speed mainly via the factor force at comparatively low speed, whereas electric drives, apart from special designs (torque motors), generate power mainly via the factor speed at comparatively low force. Since the speed is then often far higher than required on the load side of the application, reduction gears with a transmission ratio $i$ are usually introduced into the drive train. This measure apparently only

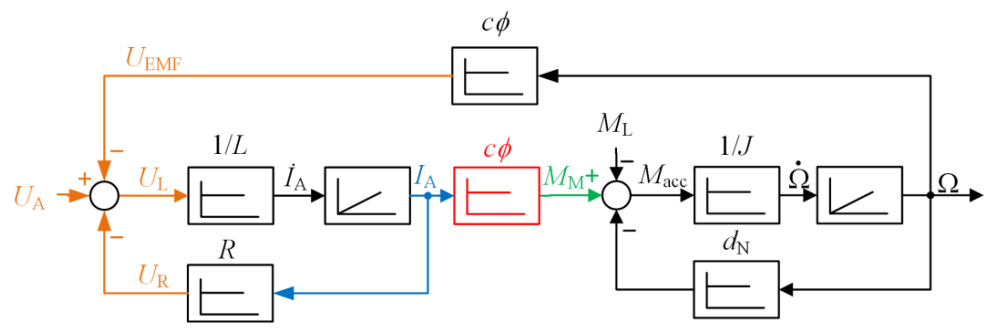

Figure 40: Block diagram of the DC drive. 
mechanically relevant has considerable control engineering consequences. The inertial mass of the load $J_{\mathrm{L}}$ is added to the total inertial mass to be driven by the motor $J_{\mathrm{M}}$ only with a factor of $1 / i^{2}$ as reduced moment of inertia. At the usual transmission ratios (usually $i>10$ ), the driven load mass changes the eigenfrequency of the drive train only marginally, whereas in the case of hydraulic drives without gears it is fully taken up, see equations in Figure 41 (index $\mathrm{M}=$ motor, $\mathrm{L}=$ load).
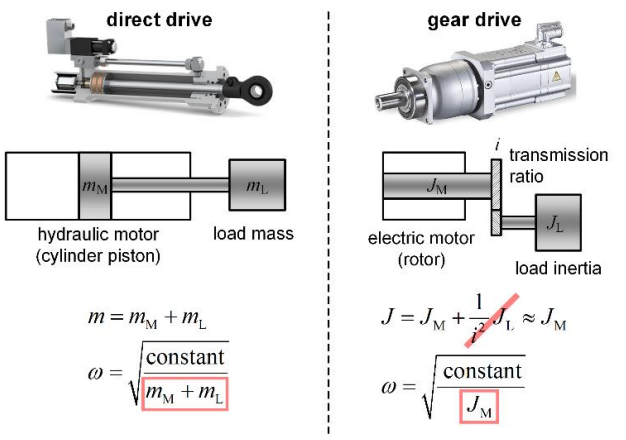

Figure 41: Mass influence on eigenfrequency.

Since the controller parameterization is significantly dependent on the eigenfrequency of the controlled system, the consequence of this fact is that the controller parameterization of a servo axis in the electrical case can be carried out without any knowledge of the load mass, whereas in the hydraulic case the controlled system can often only be optimized at the customer's system.

Due to the typical parameters of electric motors suitable for servo operation, the electrical time constant $T_{E}$ is usually many times smaller than the mechanical time constant $T_{\mathrm{M}}$, i.e. the electric motor as the possibly oscillating second-order system can be simplified to a non-oscillating first-order system in the model. If, as in the case of the compact drives mentioned above, the fixed displacement pump is driven by a variable-speed servo drive, the latter can be regarded as the actual actuator of the entire drive system whose (only) task is to generate a desired speed $\Omega_{\text {ref }}$ as fast and error-free as possible. The variablespeed drive itself is here a closed mechatronic subsystem consisting of the motor, the required encoder and current sensors, the power output stage and necessarily the implicitly required PI control loops for current (torque) and speed. This actuator subsystem can under the precondition of a well-adapted controller be regarded as a dynamic first-order system again with a specific time constant $T_{\mathrm{SD}}$. This second reduction of order is legitimized by the fact that, due to the cascade control normally used in electric servo drives, the outer speed control loop is slower than the inner current control loop and thus is the dominant link in the chain $\Omega_{\text {ref }} \rightarrow \Omega_{\text {act. }}$ If this electric servo drive is integrated into the overall hydraulic system as the actuating element, a further control loop is required for the actual controlled variable, for example the cylinder position $x$ [1], [2], see Figure 43 and Figure 44.

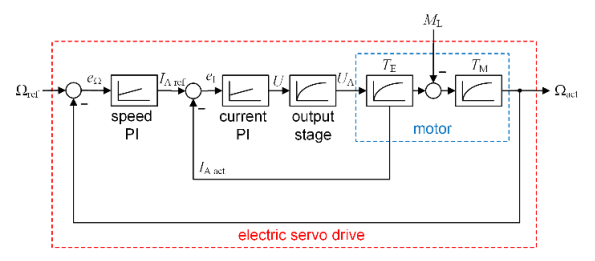

Figure 43: Simplified block diagram of the controlled electric servo drive.

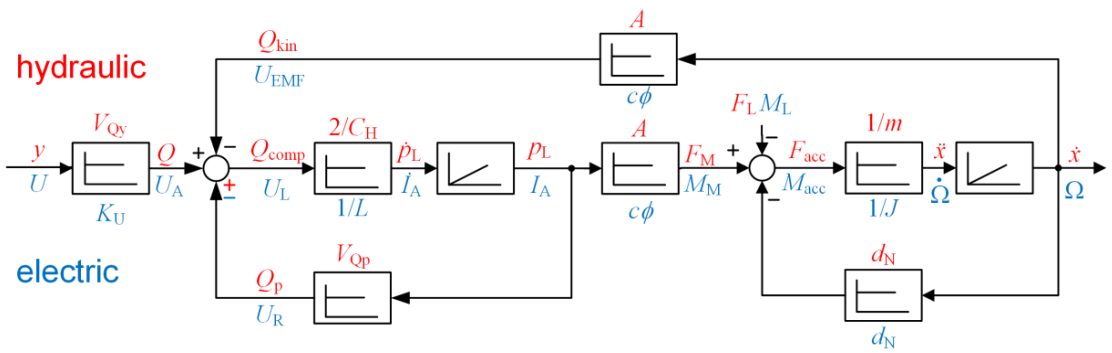

Figure 42: Block diagram of the DC drive and the idealized valve-controlled cylinder drive. 


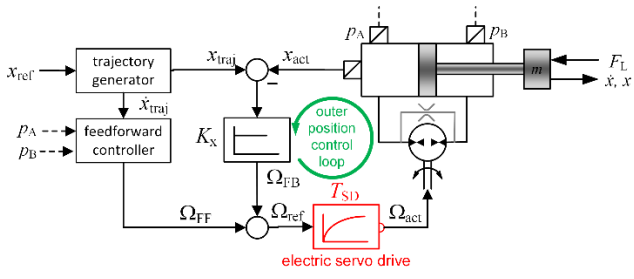

Figure 44: Use diagram of the electric servo drive as the actuating element for a pump-controlled cylinder drive.

For this outer control loop, a simple P-controller is usually sufficient here which continuously "corrects" the reference velocity for the electric servo drive. Additional model-based feedforward controllers are also possible and conceivable, of course. It should be mentioned in this context that, especially at slow speeds or when the cylinder is at a standstill, the speed of the electric drive is usually not zero, which is mainly due to the significant leakage within the pump in these operating conditions. These effects could in turn be identified and corrected with the characteristic map based control and optimization methods mentioned straight forward, whereas the desired cylinder speed and the measured pressures are the necessary input quantities.

\section{THE ROLE OF COMPUTERS}

The explanations in this paper show that the design and calculation of servo-hydraulic drive systems can be carried out systematically and purposefully. At the same time, however, it also became clear that due to the peculiarities of hydraulics, which are caused, for example, by asymmetries and non-linearities, the corresponding mathematical calculations and relationships are often not trivial. For this purpose, the possibilities of the computer-aided calculation and simulation tools available today should be used sensibly. The authors' view is that these tools should neither be used blindly nor over-automated, or that they degenerate into an end in themselves. This means that these tools are seen as pure aids $(\mathrm{CAE}=$ Computer Aided Engineering), but not as tools that release the engineer from his core tasks, i.e. the analysis and solution of corresponding problems. All too often, overflowing simulation programs are created that may very well reflect the real system behaviour, but do not really allow a true insight into the system's inherent relationships. A simple look at a chamber pressure diagram, a characteristic map or a transfer function can often produce an "aha!" effect to explain and understand the phenomena observed in reality. Such a manufacturer-independent and mathematically oriented fluid-mechatronic toolbox tool is currently being created by the authors as a Matlab Toolbox.

Nevertheless, current development trends in model-based engineering do offer possibilities for controller design that were unthinkable just a few years ago. In simulation environments, for example, controller designs can be tested and then implemented directly on the target hardware at the push of a button using appropriate code generation tools. Recently, this has even become largely platform- and manufacturer-independent (e.g. keyword FMI = Functional Mockup Interface [13], [14]). Due to the memory capacity and real-time computing performance available today and in the future, there are virtually no limits to the engineer and the possibilities associated with it.

Due to the aforementioned principle-based properties of hydraulics, this computer-based tool chain, supporting the engineer from design to implementation, seem to be made for help catapulting servo-hydraulic drive technology into the digital $21^{\text {st }}$ century. Nevertheless, a sound knowledge of mathematics, physics and technology must still be the basis for the meaningful application of these techniques.

\section{CONCLUSION AND OUTLOOK}

This paper has examined a number of current aspects of hydraulic servo drives in more detail. Although the individual points may appear somewhat heterogeneous at first glance, they all convey the same message: hydraulic drive technology may be a niche discipline, but in the mechatronic toolbox for building high level technical solutions it is indispensable and offers many unexpected perspectives. However, it can only take on and fill this important role in drive technology if it no longer considers itself as an independent discipline but defines itself rather as a fluid mechatronic part of Industry 4.0. In order 
to be competitive in the future, it is only necessary to make consistent use of the available opportunities. What is needed are not new revolutionary ideas and devices, what it needs is a new self-image. Hydraulic drive technology is the ideal solution for many tasks with high demands on motion sequences, especially in the higher power range, such as drive solutions for multi-body systems or with load masses with their own dynamics. This new self-image requires an interdisciplinary new start or better a complete reset in engineering education, where at present the signs point more to retreat than to reorientation.

\section{NOMENCLATURE}

$\begin{array}{ll}A & \text { area } \\ \alpha & \text { area ratio } \\ c & \text { electric motor constant } \\ C_{h} & \text { hydraulic capacity } \\ \mathbf{C M} & \text { characteristic map (lookup table) } \\ G & \text { transfer function } \\ d & \text { friction coefficient } \\ D & \text { damping } \\ e & \text { control error } \\ F & \text { force } \\ i & \text { gear ratio } \\ K & \text { system gain, controller gain } \\ m & \text { mass } \\ \text { OP } & \text { operating point } \\ p & \text { pressure } \\ \phi & \text { magnetic flux } \\ Q & \text { volume flow } \\ u & \text { control signal } \\ U & \text { voltage } \\ V & \text { system gain, volume } \\ x & \text { position } \\ \dot{x} & \text { velocity } \\ y & \text { valve opening, control signal } \\ \omega & \text { eigenfrequency } \\ \Omega & \text { angular velocity } \\ & \end{array}$

\section{REFERENCES}

[1] Anders P (2019) Elektrohydraulische Industrieantriebe. Lecture Notes, IFD, TU Dresden

[2] Anders P, Ströbel S (2019) Servomechanismen. Lecture Notes, Faculty of Industrial Technologies, Hochschule Furtwangen University

[3] Lutz H, Wendt W Taschenbuch der Regelungstechnik, mit MATLAB und Simulink. EuropaLehrmittel Haan
[4] Lunze J (2016) Regelungstechnik 1: Systemtheoretische Grundlagen, Analyse und Entwurf einschleifiger Regelungen. Springer Vieweg

[5] Ströbel S (2018) Modellbasierte Vorsteuerung ventilgesteuerter hydraulischer Linearantriebe im Lageregelkreis. Dissertation, TU Dresden

[6] Wey T, Lemmen M (1997) Flachheitsbasierte Regelung - Folgeregelung eines hydraulischen Differentialzylinders. Forschungsbericht Nr. 11/97 MSR, Universität Duisburg

[7] Helmke M, Ströbel S, Anders P, Schulze T. (2018) Computer-assisted modeling and automatic controller adjustment for hydraulic drives based on an innovative nonparametric identification method. 11th International Fluid Power Conference. March 19-21, 2018, Aachen, Germany

[8] Anders P, Ströbel S (2019) Regelungstechnik. Lecture Notes, Faculty of Industrial Technologies, Hochschule Furtwangen University

[9] Anders, P, Scheidt M, Bauer F (2017) Applikationsabhängig anpassbar - Antriebskonzept bietet Lösung bei Problemkonstellationen von Linearachsen. In: Antriebstechnik, Nr. 1-2, pp. 32-34

[10] Kolks G, Weber J (2018) Single Rod Cylinders with Variable Piston Area? A Comprehensive Approach to the Right Solution. In: Proceedings of the ASME/BATH Symposium on Fluid Power \& Motion Control. Bath, UK

[11] Kolks G, Weber J (2018) Getrennte Steuerkanten für den Einsatz in stationärhydraulischen Antrieben. In: O+P Fluidtechnik, Nr. 6, pp. 4251

[12] Michel S, Weber J (2012) Energieeffiziente elektrohydraulische Antriebe kleiner Leistung. Abschlussbericht zum Vorhaben TU Dresden, Institut für Fluidtechnik

[13] Modelica Association (2020): Functional Mockup Interface. https://fmi-standard.org/, Accessed 03 Feb 2020

[14] Beckhoff GmbH \& Co. KG (2020): TC3 Target for FMI. https://www.beckhoff.de/TE1420/, Accessed 03 Feb 2020 